\title{
QUALITY MANAGEMENT FOR PRESERVATION OF ANALOGUE AND DIGITAL VIDEO TAPE
}

Sebastian Gabler, NOA GmbH, Austria

\section{Formats come, formats go}

At the time of writing, Sony has just given notice on the current Betacam 1/2-inch (Beta) Video Tape Recorder (VTR) models DVW-2000P and MSW-M2000P, as the last generation of the Betacam $1 / 2$-inch (Beta) ${ }^{108}$ family, with latest orders taken by $03 / 2016$, and end of support as of 03/2023. With the first Beta-VTRs shipping in 1983, this puts an end to the Beta family after almost 40 years. The decision is significant for two main reasons. First, the Beta system had by far the highest market share of any professional VTR system, with emphasis on the Betacam SP (I986) format. ${ }^{109}$ Second, the latest $1 / 2$-inch Sony VTR will still play legacy formats, including Betacam SP, Digital Betacam, and Betacam SX. This is significant for the imminent transfer of large collections of those formats still sitting on the shelves. Beta SP machines, such as the BWV-75P, are still available as used stock and allow for a cost-efficient alternative for extraction of SP tapes.

For transferring video tapes it is required to have a good understanding of which parameters in the process chain are critical to reproduction and digitisation, in order to provide accurate preservation copies. For a Video Tape Recorder, key extraction parameters are:

- Intact tape-head system

- Provision of an accurate time base

- Composite-to-component conversion (for composite recording systems)

- Correct signal levels

All mentioned Sony VTR generations mentioned are comparatively easy to handle in migration projects. They are component-based (YUV), making any signal conversion in the digitisation process obsolete. Many Beta VTR models may be adapted to both $50 \mathrm{~Hz}$ and $60 \mathrm{~Hz}$ (PAL / NTSC) environments by the end user. All machines include Time Base Correction (TBC) that also compensates transitional drop-outs. Head Drum life is in the thousands of hours. The dominating issue with Quality Management regarding Beta family video therefore is the control of drop-outs, caused by the head-tape system.

The need for efficient transfer processes is amplified by the announced obsolescence of the Beta system. Exact figures about functionalVTRs and playable cassettes do not exist. Experience with precedent format migrations in Broadcast collections nevertheless allows for the assumption that it will not be possible to extract all holdings with the existing machines before the Beta VTR family is to be considered extinct. Typically, operating cost will rise exponentially towards the end of the usability cycle of Beta VTRs. Additionally, Beta migration projects tend to be huge-analogous to the format's historical market share - which makes cost-efficiency and Quality Management important factors.

\section{Quality Management}

Quality Assurance (QA) and Quality Control (QC) are often used as synonym terms. Under the scope of ISO 9000:2015110, they are both part of Quality Management (QM). However they have distinctly different meaning. Quality Control"I" traditionally includes item inspection. It requires the definition of quality metrics as well as the implementation of well-defined

108 http://www.sony.co.uk/pro/article/broadcast-products-vtr-announcement.

109 Schmidt, Ulrich, Professionelle Videotechnik, 6. Auflage, p. 568.

I I 0 ISO 9000:20 I5 Quality Management Systems, wnw.iso.org/iso/catalogue detail?csnumber=4548 I.

III ISO 9000:20 I5, Clause 3.3.6. 
processes, job management, skills, and experience, to name but a few. Quality Assurance ${ }^{1 / 2}$ is a superordinate instance that establishes confidence that QM criteria will be fulfilled.This implies that QC will only be executed successfully with QA in effect.

The efficiency of Quality Control can be measured as the probability to detect an error.The multiplication rule of independent events- $P(A, B)=P(A) \times P(B)$ - helps us with this problem. $P(A)$ is the error rate; $P(B)$ is the sampling rate, thus the amount of probes to be checked in a transfer process. This could involve a complete check of every $n$-th transferred item, or a partial check of every transferred item. $\mathrm{P}(\mathrm{A}, \mathrm{B})$ will, in this case, express the best possible detection rate. Obviously, this means systematic errors only need random testing to be reliably detected, but random error patterns need systematic testing, often constant testing for successful detection.

In audio-visual media, there are several initiatives dealing with $\mathrm{QC}$ metrics and methods. One prominent example is the EBU Quality Control group ${ }^{113}$ which offers several tools and resources. The scopes of Quality Control in preservation and those of access/re-use of an asset differ fundamentally. Some Archives, specifically Broadcasters, are interested in signal quality parameters to ensure a low-overhead production process, aiming at high-quality playout. Moreover, regulatory requirements may be in effect. An example for this is the requirement for the $\mathrm{BBC}^{114}$ to check programmes on Photosensitive Epilepsy Stimuli, rooted in a United Kingdom Independent Television Commission (ITC) guideline ${ }^{1 / 5}$ to protect viewers' well-being. Within a preservation process, it is however fundamental to ensure that source information has been transferred accurately, including the pre-existent deformations. Preservation is inherently sensitive to newly added defects, only.

When dealing with archival collections of video material, information frequently is not available from its instantaneous carrier. In the early years of magnetic video storage, content was copied back and forth from film to video for practical reasons in TV production. The list of obsolete video formats is long, with prominent examples including the long-serving veteran Quadruplex, which was in use from 1956 until the 1980s in professional studios. However, there are quite a few cases of obsolete systems that served for considerably shorter periods, and became swiftly extinct after their commercial obsolescence, such as the Panasonic D3 system. Analogue-toanalogue migrations were common with VTR generations passing. Often, the previous carrier generation was terminally discarded, including original film stock. Against this backdrop, in contrast to the rules applicable for audio archival content ${ }^{116}$, often the latest replication is the only accessible manifestation of certain programmes.

In the past, mass-migration was no less demanding than it is today. Replicated recordings often suffer defects originating one or several generations back. We find film scratches on IMX tapes, or defects typical for U-Matic on material played from Betacam SP.

For preservation transfers, it is important to emphasise the importance of QA as a key means of producing authentic information, and the main scope of $Q C$ here is to detect if any errors have been added in the last transfer. It is critical for efficient preservation Quality Management to provide such evidence in the Quality Check task, as otherwise any noticeable defect will raise the question of a transfer error. In an OAIS environment, Quality Management information is involved in Preservation Description Information, and is typically included when accessioning an Archival Information Package (AIP). ${ }^{17}$

II2 ISO 9000:20 I5, Clause 3.3.7.

113 https://tech.ebu.ch/groups/qc.

I 4 BBCWHP 167, D3 Preservation File Format, http://downloads.bbc.co.uk/rd/pubs/whp/whp-pdf-files/WHP|67.pdf.

I 5 http://stakeholders.ofcom.org.uk/binaries/broadcast/guidance/gn flash.pdf.

I 6 IASA TC-04 Guidelines in the Production and Preservation of Digital Audio Objects: standards, recommended practices, and strategies: 2nd edition: 5.2.2 Selection of best copy.

117 OAIS Reference Model, CCSDS 650.0-M-2, p.4-38, http://public.ccsds.org/publications/archive/650x0m2.pdf. 
With its first products released around $2000, \mathrm{NOA} \mathrm{GmbH}^{118}$ has provided quality-controlled archival transfer tools and processes for over 15 years to archives and service providers. It was part of NOA's founding story to offer automated aides that allow a more reliable Quality Management than manual methods, and that allow reducing the time requirements of Quality Control at the same time. ${ }^{119}$ The understanding is that real-time QC in audiovisual media is very time consuming, thus it becomes expensive and inaccurate (due, in part, to fatigue with the operators). Sample checking is not reliable enough, specifically not for random error patterns. That dilemma has resulted in a method called Traces Aided Spot Checking $\left(T_{A S}{ }^{120}\right)$, as a proven solution. The underlying principle of TAS is providing quality metrics as metadata, such as error rates and error events, or annotated re-player conditions such as Azimuth, ISR BLER, or C2-Errors, aligned in time with the extracted media. This data is produced automatically, without user interference. The generated information then may be used for automated or manual Quality Check procedures. This may happen, for example, by counting the occurrence of certain events such as ISR errors in a certain transfer, or helping a quality control operator to identify questionable portions of the signal for checking, as examples in this paper will highlight.

\section{VTR drop-out detection}

Typical playback interferences, such as dirt and loss of information in the magnetic layer, misalignment, clogging, and wear of the reproduction heads, manifest in transient or permanent loss of carrier signal level. The A/V Artifact Atlas project has undertaken to make examples of such defects publically available. ${ }^{21}$ These problems are colloquially called "drop-out." In an analogueVTR they usually manifest themselves in line-based artefacts, whereas digital video usually exhibits block-based deformations. In extreme cases, for example, when the tape coating has disintegrated, or shed tape coating has clogged the video heads, the entire signal is lost, and complete break-up will occur.

Drop-outs may be detected using various approaches. Essence-based drop-out detection aims at finding typical patterns in the information (that is, in the coded picture), hinting at a problem. This usually happens in the digital domain. Pattern detection requires considerable computing resources. Its reliability depends on the accuracy of the model applied.Therefore, each specific error pattern needs explicit implementation. The main problem with preservation transfers however is that this method cannot provide the information if an error has occurred during extraction, or if it was already previously recorded. Such conclusion, without transfer quality monitoring in place, is only possible by taking assumptions from the complete genealogy of the recording. If the current media is not the instantaneous carrier, the interpretation will become complex, or a stringent assertion may even be impossible to make. This is an aspect that is relevant regardless of how accurate pattern recognition was implemented.

The carrier- and channel code based detection methods suggested here have the advantage to specifically provide information about drop-outs occuring during an extraction. They work independent of the actual error pattern in the essence, on a higher protocol layer than essence based detection. Carrier based detection measures the RF carrier amplitude periodically accumulated with the frame rate. The amplitude subsequently is statistically analysed, and compared to the expectation value. Thus, an error pointer is provided to the user, pointing at ranges with a high likelihood of a transfer-related signal error, as required for Traces Aided Spot Checking.

$118 \mathrm{http}: / /$ noa-archive.com

I 19 https://web.archive.org/web/20030629072430/http://www.noa-audio.com/philosophy.html.

I 20 Sebastian Gabler, in 24th TONMEISTERTAGUNG -VDT INTERNATIONAL CONVENTION, November, 2006, p. $527 \mathrm{ff}, 3.3$.

121 http://avaa.bavc.org/artifactatlas/index.php/AN Artifact Atlas. 


\section{Drop out detection on Beta SP}

All analogue VTRs use a frequency modulated (FM) luminance signal.There are two main reasons for that, which both root in the characteristics of magnetic tape. Frequency Modulation shifts the lower limit upwards to approximately $2 \mathrm{MHz}$. Thus, relative bandwidth is reduced to 3-4 octaves. This removes the requirement to compensate for tape's reduced response at low frequencies. FM signals encode information by variation of the instantaneous frequency. FM encoded information is immune to level attenuation, distortion, and noise disturbance. Transmission reliability will be greatly enhanced by clipping the signal to tape-saturating levels. With Beta SP, the resulting bandwidth for the luminance FM signal is $6.8-8.8 \mathrm{MHz}$.

Figure I illustrates that the best measurement conditions for the purpose are to be found after the VTR's reproduction circuit, before the Time Base Correction (TBC) unit.

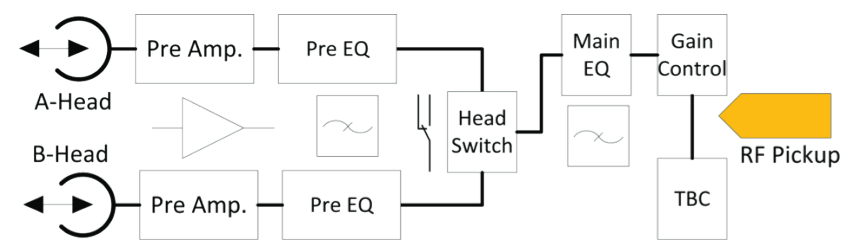

Figure I:VTR Repro Block Diagram.

NOA has developed specific hardware to be attached directly on the VTR's reproduction circuit board (Sensor Plate), and connected to a processing device (name: VTRi). That device also includes VTR remote control interface and transmits QC metadata to NOA's proprietary Video capturing software, for two VTRs in parallel. The entire detection process spans across three main steps.

\section{I Measurement}

After buffering the RF signal, the logarithmic, rectified envelope is generated. This is an analogue signal processing step. As displayed in Figure 2, the relevant signal characteristics are already apparent by an analogue measurement, using a standard vector scope.
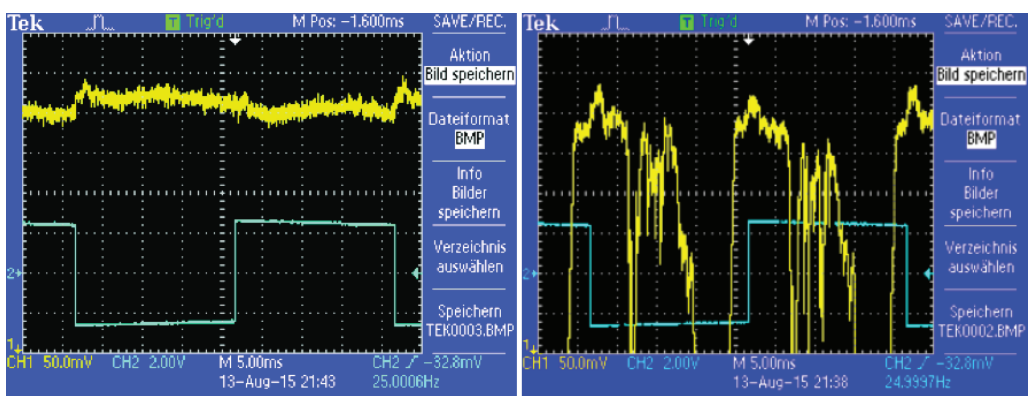

Figure 2: Normal RF envelope vs. Drop-out pattern

\subsection{Sampling}

Using an Analog to Digital Converter (ADC), the envelope is sampled at a rate of $4000 \mathrm{~Hz}$. This results in 80 values per field for PAL, and 66-67 values for NTSC.The design thus provides sufficient precision to locate a defect within a given video frame, with robust reliability. 


\subsection{Evaluation}

The evaluation is divided into two main steps. First, a frequency table ${ }^{122}$ is produced per evaluation period. A frequency table is a tool used in Statistics to model the distribution of values by the frequency of their occurrence. In this case, we quantise the RF level into an evaluation range, divided into equidistant groups. These data are sent to the recording software with a time-stamp of the respective period. The sample window defaults to $25 \mathrm{~ms}$, but may be adjusted to 16 ms for NTSC field equivalent. Subsequently, the frequency table is processed with a specific weighting model, rendering a dimensionless error-coefficient $m$.

$$
m=\frac{\sum M_{w}}{n}
$$

Coefficient $m$ provides the key quality indicator used for traces and quality event information. The weighing model takes into account that the typical (healthy) distribution will be grouped narrowly around the maximum count with most values in a range of app. +-2 value groups. The highest count will be towards the $0 \mathrm{~dB}$ margin, which relates to the nominal RF modulation level, see Figure 3 below. The weighing model returns a low error coefficient value for healthy signals.

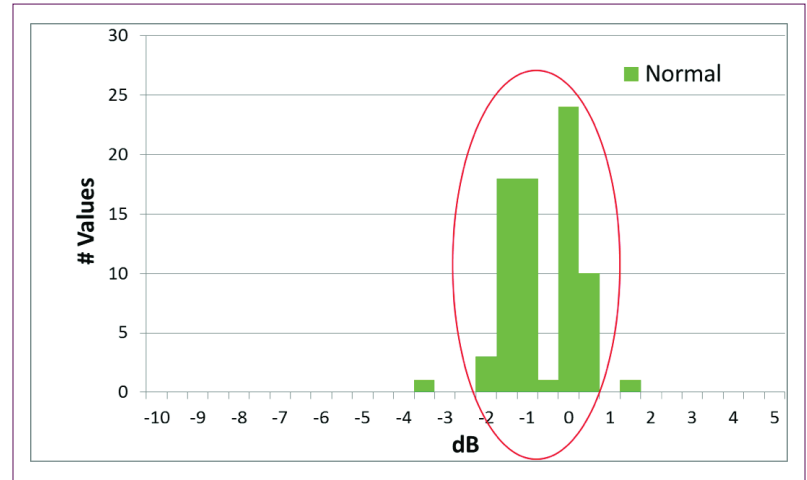

Figure 3:Value distribution for healthy signal.

In the case of an error, values down to the low end of the evaluation range exist.Values will be distributed between the low border of the evaluation range and the maximum count group. The weighing model takes these patterns into account, rendering a high error coefficient value. See Figure 4 below for an example.

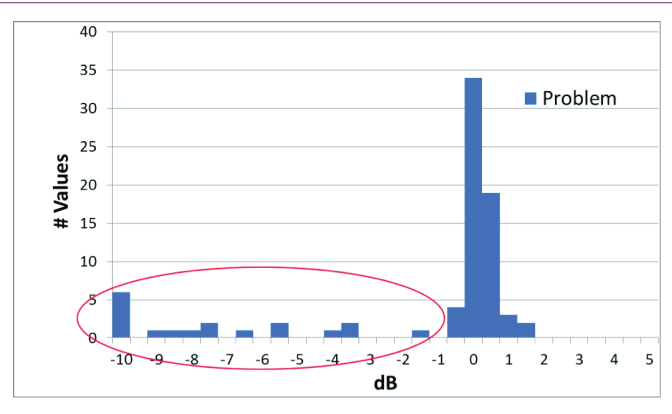

Figure 4:Value distribution for Drop Out. 
Note that Figure 3 and Figure 4 are taken from the same experiment that provided the vector scope shots from Figure 2. The direct correlation of both visualisations becomes obvious. Figure 5 illustrates how a defect is presented in the capturing software to the user. The active frame shows a multi-line drop-out, in place with a highlighted error event marker ( $m$ below error threshold).

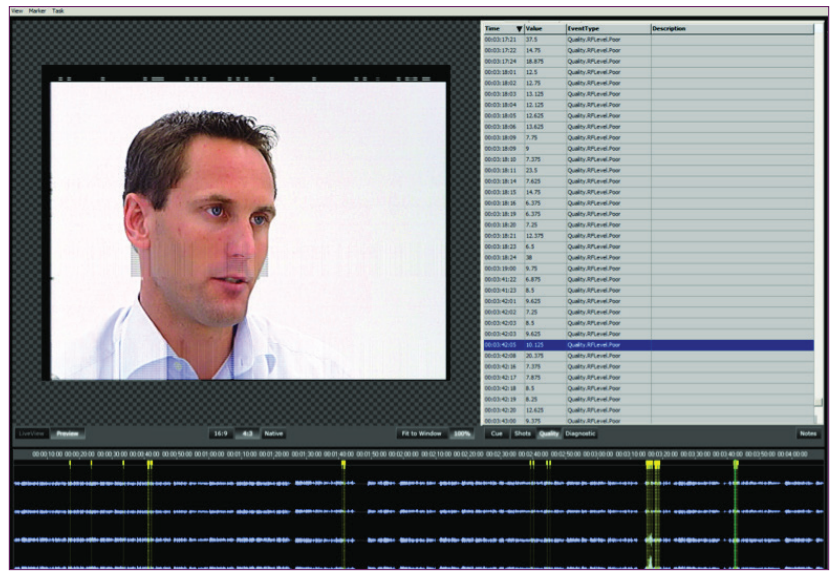

Figure 5: Beta SP Line Artefact.

On the other hand, when validating the problem shown in Figure 6, the low coefficient value ( $m$ is I.8 .. 2.3) of the RF trace clearly identifies the drop-out as a pre-existent part of the signal. Quality Control thus may assert that a re-transfer of this medium would be futile.

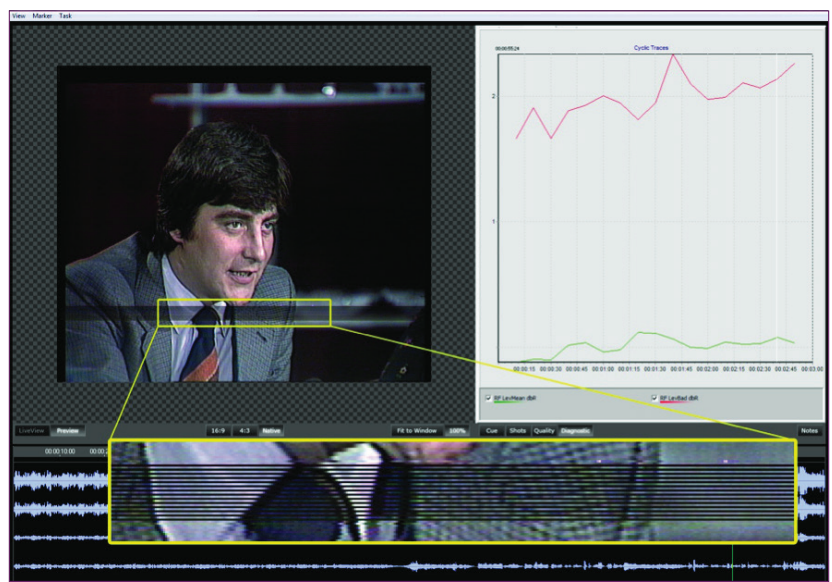

Figure 6: Burned-in defect. 


\section{Drop out control on digital VTRs}

Digital Beta family VTRs include built-in systems for status reporting. These systems can be made usable for the QA of archival transfer processes.

ISR, or Interactive Status Reporting, is a protocol aiming at the remote monitoring, diagnostics, and maintenance of broadcast equipment, proprietary to Sony. Based on the SMPTE standard Status Monitoring and Diagnostics Protocol (SMDP, SMPTE 273M), ${ }^{123}$ ISR features a private SMDP command set that includes functions suited for runtime signal monitoring. Sony has implemented the protocol in several generations of their professional VTRs, including professional DAT machines, Digibeta, and IMX families. Machines that support ISR bear the ISR Logo.

Details of the ISR specification are not publically available. However, copies of the original Sony documentation can be obtained. Machines supporting ISR have a dedicated RS232 port, which interfaces with a Data Terminal. ${ }^{124}$ To make the ISR data available for use with the capturing software, NOA provides the same VTR-i hardware as for RF measurements. The connectivity is again 2 VTRs per VTR-i.

For the purpose of tape playback monitoring (LEVEL NAME PLAY ${ }^{125}$ ), ISR offers both transient and cyclic registers. Events are maintained per audio and video track and labelled as "Channel Condition". Sony specifies five event levels $(0$ ="good" | I="almost good" | $2=$ ="indeterminable" | 3="doubtful" | 4="no good"). The cyclic register is supposed to store any condition events different than level 0 , since the last query, together with their occurrence time code. ${ }^{126}$ However, the authors have consistently experienced that this register misses any events classified as level I, and reports all events of levels $2-4$ as being level 4 events. The content of the transient register returns accurate information. That however is transitory in the field rate of the signal and therefore needs continuous refresh.

At the same time, ISR machines offer a Channel Condition traffic light LED (known in industry as "Lamp") on the front panel, displaying a green light to report normal operation conditions, yellow light for "somewhat deteriorated" and red light to report playback errors. In line with the specification in the MSW2000 user manual ${ }^{127}$ about the Channel Conditions traffic lights, ISR status flags 4 correlate with red LED signal.This falls together with the observation that ISR events from analogue tapes are only flagged with 0 and 4; hence ISR is not sufficiently accurate for the use with analogue tapes.

Used with digital tape formats, ISR results however are accurate. Figure 7 exemplifies image break-up resulting from a tape coating damage. Enlarged, one significant block artefact should reproduce well in printing. The quality problem is again in line with the highlighted error event in the marker side-panel. Ongoing experience with Digibeta transcriptions, for example, shows that ISR reliably reports any blocking artefacts, regardless of the pattern. Therefore, the detection method is universally usable for all digital formats.

123 http://ieeexplore.ieee.org/xpl/articleDetails.jsp?arnumber=7292091.

124 http://www.ibm.com/support/knowledgecenter/ssw aix 53/com.bm.aix.commadmn/doc/commadmndital asynch modems dataterm.htm.

125 Sony, ISR Protocol, Sony document number 9-967-6/4-0 I, p. 59 f.

126 lbidem.

127 "During analog playback indications are by red and green only," MSW-*2000* Operation Manual Ist edition (Revised 2) p. 2-7. 


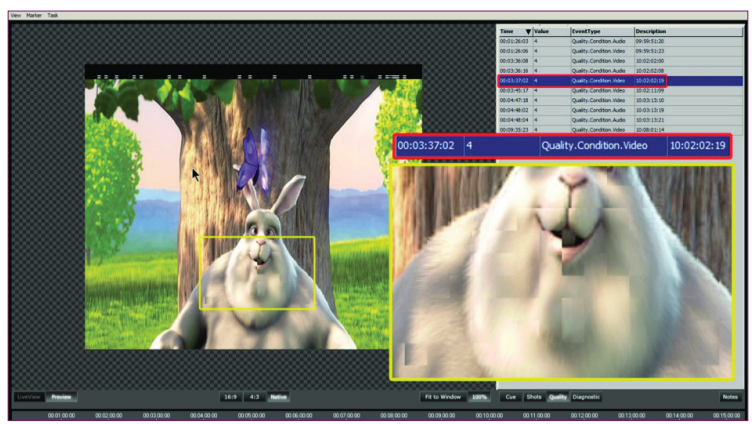

Figure 7: Digital Drop Out on IMX.

\section{Conclusions and outlook}

Monitoring drop-outs continuously during transfer yields a very high level of Quality Management for archival transfers of video tape. Maintaining correct operating conditions, and being able to trace these is fundamental to the Quality Assurance of such a process. The error pointers generated from RF envelope processing and ISR monitoring enable efficient Quality Check procedures for the operators, and help to identify actual transfer problems, and allow for discrimination of them from burnt-in defects.

The Quality Management approach discussed in this article refers to analogue Beta SPVTRs as well as to DigiBeta and IMXVTRs, but with a different scope. Whereas the aspects relevant to analogueVTRs are developed specifically for the Beta SP format, the applied principles are applicable within certain constraints to any analogueVTR, beginning with FM-Direct systems like the original Quadruplex.The methods supporting ISR have only been tested in use with Sony products, even if Panasonic D3 VTRs seem to have employed similar status reporting tools. ${ }^{128}$ That being said, any D2, D3, and D5VTRs of Ampex and Panasonic are basically extinct, which excludes them as a subject of large-scale migration projects these days. Controlling drop-outs does not cover all possible bases of VTR related Quality Management; for example incorrect signal levels during extraction may lead to information loss in preservation. With excessive levels, clipping of the AD converters may lead to distortion artefacts. De-rated levels may incur additional noise in later processing, specifically when using 8-bit quantisation. Going into the details of component signal levels would however exceed the scope of this paper, and in specific context with broadcastgrade BetaVTRs, there is not so much focus on these aspects as usually signal level management may be assumed as efficient.

Finally, the monitoring and logging of drop-out rates also opens new perspectives for equipment maintenance. IMXVTR heads are rated with 6,000 operating hours. As any mechanically wearable part, its imminent failure will be signalled by an exponential growth of the error events. Using the long-term statistics of drop-out rates, a base line is determined for every individual device, and any change against that base line. Taking into account that the maintenance of an IMX machine costs about I Euro per operating hour, and that spare parts are only available for a limited time now, any maintenance that aims at leveraging wearable parts beyond their nominal lifetime has substantial efficiency potential for Archives and Service Providers. When doing so, efficient process Quality Management comes as a valuable safety net.

\section{Acknowledgments}

The author would like to thank Christoph Gerstbauer and Marcus Krämer for their valuable contributions. Some sample material for measurements (Figure 6) has been kindly commissioned by Slovak Radio Television, RTVS, Bratislava. 